\title{
INVARIANT PARALLELS, INVARIANT MERIDIANS AND LIMIT CYCLES OF POLYNOMIAL VECTOR FIELDS ON SOME 2-DIMENSIONAL ALGEBRAIC TORI IN $\mathbb{R}^{3}$
}

\author{
Jaume Llibre and Salomón Rebollo-Perdomo \\ Departament de Matemàtiques, \\ Universitat Autònoma de Barcelona, \\ 08193 Bellaterra, Barcelona, Catalonia, Spain
}

E-mail address: jllibre@mat.uab.cat, srebollo@mat.uab.cat

\begin{abstract}
We consider the polynomial vector fields of arbitrary degree in $\mathbb{R}^{3}$ having the 2-dimensional algebraic torus$$
\mathbb{T}^{2}(l, m, n)=\left\{(x, y, z) \in \mathbb{R}^{3}:\left(x^{2 l}+y^{2 m}-r^{2}\right)^{2}+z^{2 n}-1=0\right\},
$$

where $l, m$ and $n$ positive integers, and $r \in(1, \infty)$, invariant by their flow. We study the possible configurations of invariant meridians and parallels that these vector fields can exhibit on $\mathbb{T}^{2}(l, m, n)$. Furthermore we analyze when these invariant meridians or parallels are limit cycles.
\end{abstract}

\section{InTRODUCTION AND STATEMENT OF THE MAIN RESUltS}

In 1878 Darboux published two works, [5] and [6], about polynomial vector fields or equivalently autonomous polynomial differential equations on $\mathbb{R}^{n}$ or $\mathbb{C}^{n}$. There, he showed that if a polynomial vector field has a sufficient number of invariant algebraic hypersurfaces then it has a first integral. If we have a polynomial vector field in $\mathbb{R}^{n}$ or $\mathbb{C}^{n}$ with a first integral, then we can reduces its study in one dimension; of course, in the planar case we can describe completely its phase portrait, which is the main goal of the qualitative theory of differential equations.

Darboux's work was improved by Jouanolou [12] in 1979, and recently in [18] the authors improve the Darboux's classical result and the new one of Jouanolou taking into account the multiplicity of the invariant algebraic hypersurfaces. Since Darboux's result, it has existed a big interest in the study of invariant algebraic hypersurfaces of polynomial vector fields, in particular in the planar case (see for instance [3,4] and references there in). Recently several papers have been published looking for particular invariant algebraic sets, for instance invariant straight lines (see $[22,21,20,1,15])$, invariant hyperplanes in $\mathbb{R}^{n}$ (see $[17,13]$ ), invariant circles and limit cycles for polynomial vector fields on the sphere (see $[2,10,16])$.

The study of limit cycles of polynomial vector fields on the plane has been intensively treated since 1900 due to the second part of the 16-th Hilbert problem, which ask about the configuration, i.e. the number and relative position, of the limit cycles that a planar polynomial vector field of degree $d$ can exhibit. In particular, a part of this problem is:

2010 Mathematics Subject Classification. 34C05, 37C10, 37C27.

Key words and phrases. polynomial vector fields, invariant parallel, invariant meridian, limit cycles, periodic orbits, 2-dimensional torus. 
Provide an upper bound, depending only on $d$, for the maximum number of limit cycles that any polynomial vector field of degree $d$ can have, for more details see $[8,9,11]$.

In this work we consider the 2-dimensional algebraic torus

$$
\mathbb{T}^{2}(l, m, n)=\left\{(x, y, z) \in \mathbb{R}^{3}:\left(x^{2 l}+y^{2 m}-r^{2}\right)^{2}+z^{2 n}-1=0\right\},
$$

where $l, m$, and $n$ are positive integers, and $r$ is a real number greater than one. We are going to study the polynomial vector fields of arbitrary degree in $\mathbb{R}^{3}$ having the 2dimensional algebraic torus $\mathbb{T}^{2}(l, m, n)$ invariant by their flow. We will study the possible configurations of invariant parallels and meridians that these vector fields can exhibit on $\mathbb{T}^{2}(l, m, n)$. Additionally we will consider when these invariant parallels or meridians can be (algebraic) limit cycles. Our goal is to generalize to all $l, m$ and $n$ the results given in [14] where the authors consider the particular case $l=m=n=1$.

On $\mathbb{T}^{2}(l, m, n)$ we define parallels and meridians as the curves obtained by the intersection of this algebraic surface with the planes orthogonal to the $z$-axis and the planes containing the $z$-axis, respectively. More precisely, a parallel of $\mathbb{T}^{2}(l, m, n)$ is a connected component of the intersection $\left\{z-z_{0}=0\right\} \cap \mathbb{T}^{2}(l, m, n)$, with $z_{0} \in[-1,1]$. If $z_{0} \neq \pm 1$ such intersection has two parallels, otherwise it has only one parallel. A meridian of $\mathbb{T}^{2}(l, m, n)$ is a connected component of the intersection $\{a x+b y=0\} \cap \mathbb{T}^{2}(l, m, n)$, with $a, b \in \mathbb{R}$ and $a^{2}+b^{2} \neq 0$. So meridians always are in pairs.

If a polynomial vector field $\mathcal{X}$ in $\mathbb{R}^{3}$ has the algebraic torus $\mathbb{T}^{2}(l, m, n)$ invariant by its flow, then a parallel of $\mathbb{T}^{2}(l, m, n)$ is called invariant of $\mathcal{X}$ if it is formed by orbits of $\mathcal{X}$. In similar way we define invariant meridian. We note that a parallel or a meridian which is invariant of $\mathcal{X}$ is an invariant algebraic curve of $\mathcal{X}$.

Let $\mathfrak{X}_{d}(l, m, n)$ be the set of polynomial vector fields of degree $\leq d$ having $\mathbb{T}^{2}(l, m, n)$ invariant by their flow. The set $\mathfrak{X}_{d}(l, m, n)$ is a $\mathbb{R}$-vector space of finite dimension. If $\mathfrak{X}_{d}(l, m, n)$ is different from the zero polynomial vector field, then we can identify it with the affine space $\mathbb{R}^{q}$ for some $q \geq 1$, by associating to each element $\mathcal{X}$ of $\mathfrak{X}_{d}(l, m, n)$ a point of $\mathbb{R}^{q}$ whose coordinates are all the different free coefficients of $\mathcal{X}$. We can give to $\mathfrak{X}_{d}(l, m, n)$ the topology induced by this identification. Thus we say that a subset of $\mathfrak{X}_{d}(l, m, n)$ is generic if its image in $\mathbb{R}^{q}$ is an open and dense subset.

In our study we can assume that $l \geq m$, in otherwise we take the change of coordinates $(x, y, z) \rightarrow(y, x, z)$ to obtain the desired condition.

Our main result about the maximum number of invariant parallels and invariant meridians is the following.

Theorem 1. Suppose that $\mathcal{X} \in \mathfrak{X}_{d}(l, m, n)$ has finitely many invariant parallels and invariant meridians.

(a) If $2 n \geq 2 l+1$ then the maximum number of invariant parallels of $\mathcal{X}$ is $2(d-2 l-1)$, and the maximum number of invariant meridians of $\mathcal{X}$ is $2(d-2 l+1)$. Moreover there are $l, m, n, d$ and $\mathcal{X} \in \mathfrak{X}_{d}(l, m, n)$ such that $\mathcal{X}$ has either $2(d-2 l-1)$ invariant parallels, or $2(d-2 l+1)$ invariant meridians.

(b) If $2 n<2 l+1$ then the maximum number of invariant parallels of $\mathcal{X}$ is $2(d-2 n)$, and the maximum number of invariant meridians of $\mathcal{X}$ is $2(d-2 n+2)$. Moreover there are $l, m, n, d$ and $\mathcal{X} \in \mathfrak{X}_{d}(l, m, n)$ such that $\mathcal{X}$ has either $2(d-2 l-1)$ invariant parallels, or $2(d-2 l+1)$ invariant meridians.

The study of the distribution of invariant parallels and invariant meridians of $\mathcal{X} \in$ $\mathfrak{X}_{d}(l, m, n)$ for arbitrary $l, m, n$ and $d$ is a difficult problem. Hence in order to obtain 
satisfactory results we need to consider some restrictions on $l, m, n$ and $d$. A first result in this direction is the following where $l, m$ and $n$ have arbitrary values, but we consider a restriction on the degree of the vector fields.

Theorem 2. If $d<2 l-1$ then the following statements hold.

(a) If $\mathcal{X} \in \mathfrak{X}_{d}(l, m, n)$ and $d<2(m+n)-1$, then $\mathcal{X}=0$.

(b) If $\mathcal{X} \in \mathfrak{X}_{d}(l, m, n), \mathcal{X} \neq 0$, and $d \geq 2(m+n)-1$, then $\mathcal{X}$ has finitely many invariant parallels and invariant meridians. Moreover,

(b.1) $\mathcal{X}$ has at least two and at most $2(d-2(m+n)+2)$ invariant parallels.

(b.2) $\mathcal{X}$ has at least two and at most $2(d-2(m+n)+2)$ invariant meridians.

(b.3) The sum of invariant parallels and invariant meridians of $\mathcal{X}$ is at least four and at most $2(d-2(m+n)+3)$.

(c) If $\mathcal{X} \in \mathfrak{X}_{d}(l, m, n)$ then, any invariant parallel or invariant meridian of $\mathcal{X}$ is not a limit cycle.

(d) For every $\nu \in\{0,1, \ldots, 2(d-2(m+n)+1)\}$ and every $k \in\{0,1, \ldots, d-2(m+n)+1\}$ such that $\nu+2 k \leq 2(d-2(m+n)+1)$, there exists $\mathcal{X} \in \mathfrak{X}_{d}(l, m, n)$ having exactly $\nu+2$ invariant parallels and $2(k+1)$ invariant meridians.

Under the condition $d<2 l-1$ Theorem 2 gives a complete description about all the possible configurations of invariant parallels, invariant meridians, and shows that they cannot be limit cycles.

The following two results generalize to the case $l=m=n$ the main results of [14].

Theorem 3. Assume that $\mathcal{X} \in \mathfrak{X}_{d}(n, n, n)$ has finitely many invariant meridians and invariant parallels.

(a) $d \geq 2 n-1$.

(b) The maximum number of invariant parallels of $\mathcal{X}$ is $2(d-2 n)$.

(c) The maximum number of invariant meridians of $\mathcal{X}$ is $2(d-2 n+1)$.

Theorem 4. Let $k \in\{0,1, \ldots, d-2 n+1\}$ and $\nu \in\{0,1, \ldots, 2(d-2 n)\}$ be.

(a) There exists $\mathcal{X} \in \mathfrak{X}_{d}(n, n, n)$ having exactly $\nu$ invariant parallels and $2 k$ invariant meridians.

(b) There exists $\mathcal{X} \in \mathfrak{X}_{d}(n, n, n)$ having exactly either $\nu$ invariant parallels or $2 k$ invariant meridians which are algebraic limit cycles.

Theorems 3 and 4 give all the possible configurations of invariant parallels and invariant meridians and show when they are limit cycles.

At the moment we cannot be able to provide complete results as above for arbitrary $l$, $m, n$ and $d$; however under generic conditions on the vector fields on $\mathbb{T}^{2}(l, m, n)$ and some restrictions on $l, m, n$ and $d$ we can give all the possible configurations of invariant parallels and invariant meridians and show when they are limit cycles, the result is the following.

Theorem 5. Let $d, l, m$ and $n$ be positive integers such that either $d \geq 2 n \geq 2 l$ if $l=m$, or $d \geq 2 n \geq 2(l+m)$ if $l>m$. There exists a generic subset $\mathcal{G} \subset \mathfrak{X}_{d}(l, m, n)$ such that:

(a) If $\mathcal{X} \in \mathcal{G}$ then the maximum number of invariant parallels of $\mathcal{X}$ is $2(d-2 n)$.

(b) If $\mathcal{X} \in \mathcal{G}$ then the maximum number of invariant meridians of $\mathcal{X}$ is $2(d-2 l+1)$.

(c) For every $k \in\{0,1, \ldots, d-2 l+1\}$ and every $\nu \in\{0,1,2, \ldots, 2(d-2 n)$ there exists $\mathcal{X} \in \mathcal{G}$ having exactly $\nu$ invariant parallels and $2 k$ invariant meridians.

(d) For every $k \in\{0,1, \ldots, d-2 l+1\}$ and every $\nu \in\{0,1,2, \ldots, 2(d-2 n)$ there exists $\mathcal{X} \in \mathcal{G}$ having exactly either $\nu$ invariant parallels, or $2 k$ invariant meridians which are limit cycles. 
The paper is organized as follows. In Section 2 we will give the necessary definitions about invariant algebraic surfaces of polynomial vector fields in $\mathbb{R}^{3}$. Also we will recall the concept and properties of the extactic polynomial, which we will be the main tool for our study. In Section 3 we are going to obtain general properties and conditions about the polynomial vector fields on $\mathbb{T}^{2}(l, m, n)$, which allow us to give the proofs of the main results. Theorems 1 and 2 will be proved in Sections 4 and 5 respectively. In Section 6 we will give the proof of Theorems 3 and 4. Finally, in Section 7 we will give the proof of Theorems 5 .

\section{VECTOR FIELDS OF DEGREE $d$}

Let $\mathbb{R}[x, y, z]$ be the ring of the polynomials in the variables $x, y$ and $z$ with real coefficients. Recall that a polynomial vector field $\mathcal{X}$ of degree $d$ in $\mathbb{R}^{3}$ is an expression of the form

$$
\mathcal{X}=P \frac{\partial}{\partial x}+Q \frac{\partial}{\partial y}+R \frac{\partial}{\partial z}
$$

where $P, Q, R \in \mathbb{R}[x, y, z]$, and $d=\max \{\operatorname{deg}(P), \operatorname{deg}(Q), \operatorname{deg}(R)\}$.

If $F \in \mathbb{R}[x, y, z]$ then the algebraic surface $\{F=0\} \subset \mathbb{R}^{3}$ is called an invariant algebraic surface of the vector field (1) if there exists $K \in \mathbb{R}[x, y, z]$ such that

$$
\mathcal{X} F=P F_{x}+Q F_{y}+R F_{z}=K F,
$$

where $F_{x}, F_{y}$ and $F_{z}$ denote the partial derivatives of $F$ respect to $x, y$ and $z$ respectively. The polynomial $K$ is called the cofactor of the invariant algebraic surface $\{F=0\}$. Moreover if $\mathcal{X}$ is of degree $d$ then the cofactor $K$ is of degree at most $d-1$. Also from (2) we get that if an orbit of (1) has a point in the algebraic surface $\{F=0\}$, then the whole orbit is contained in $\{F=0\}$, i.e. $\mathcal{X}$ has to $\{F=0\}$ invariant by its flow. This justifies the name of invariant surface.

Let $F \in \mathbb{R}[x, y, z]$ be a fixed polynomial. A polynomial vector field (1) that satisfies (2) is called a polynomial vector field on the algebraic surface $\{F=0\}$. If $\mathfrak{X}(F)$ denotes the set of polynomial vector fields (1) on $\{F=0\}$, then from (2) we get that $\mathfrak{X}(F)$ is an $\mathbb{R}$-vector space.

Now we will introduce a polynomial which will allow to detect when an algebraic surface is invariant by the flow of a polynomial vector field.

Let $\mathcal{X}$ be the polynomial vector field (1) and let $W \subset \mathbb{R}[x, y, z]$ be a $\mathbb{R}$-vector space of finite dimension $p>1$. The extactic polynomial of $\mathcal{X}$ associated to $W$ is the polynomial $\mathcal{E}_{W}(\mathcal{X})$ given by the determinant of the matrix

$$
\left(\begin{array}{cccc}
v_{1} & v_{2} & \cdots & v_{p} \\
\mathcal{X}\left(v_{1}\right) & \mathcal{X}\left(v_{2}\right) & \cdots & \mathcal{X}\left(v_{p}\right) \\
\vdots & \vdots & \cdots & \vdots \\
\mathcal{X}^{p-1}\left(v_{1}\right) & \mathcal{X}^{p-1}\left(v_{2}\right) & \cdots & \mathcal{X}^{p-1}\left(v_{p}\right)
\end{array}\right)
$$

where $\left\{v_{1}, \ldots, v_{p}\right\}$ is a basis of $W$ and $\mathcal{X}^{j}\left(v_{i}\right)=\mathcal{X}^{j-1}\left(\mathcal{X}\left(v_{i}\right)\right)$. The definition of the extactic polynomial $\mathcal{E}_{W}(\mathcal{X})$ is independent of the chosen basis of $W$ because of the properties of the determinant and of the derivation.

Example 1. Consider the polynomial vector field in $\mathbb{R}^{3}$

$$
\mathcal{X}=x y z \frac{\partial}{\partial x}+2 z\left(y^{2}+2\right) \frac{\partial}{\partial y}+4 y\left(z^{2}-1\right) \frac{\partial}{\partial z},
$$


and the subspace $W=\{1, x, y\}$. The components of $\mathcal{X}$ are $P=x y z, Q=2 z\left(y^{2}+2\right)$ and $R=4 y\left(z^{2}-1\right)$, then

$$
\mathcal{E}_{\{1, x, y\}}(\mathcal{X})=\operatorname{det}\left(\begin{array}{ccc}
1 & x & y \\
\mathcal{X}(1) & \mathcal{X}(x) & \mathcal{X}(y) \\
\mathcal{X}^{2}(1) & \mathcal{X}^{2}(x) & \mathcal{X}^{2}(y)
\end{array}\right)=\operatorname{det}\left(\begin{array}{ccc}
1 & x & y \\
0 & P & Q \\
0 & \mathcal{X}(P) & \mathcal{X}(Q)
\end{array}\right),
$$

where $\mathcal{X}(P)=P P_{x}+Q P_{y}+R P_{z}$ and $\mathcal{X}(Q)=P Q_{x}+Q Q_{y}+R Q_{z}$. A simple computation shows that

$$
\mathcal{E}_{\{1, x, y\}}(\mathcal{X})=2 x z^{3}\left(2+y^{2}\right)(y-2)(y+2) .
$$

We remark that the previous matrix already appears in the work of Lagutinskii (see Dobrovol'skii et al. [7]). For a geometric explanation of the meaning of such a matrix see Pereira [19]. Christopher et al. [4] used the extactic polynomial to study the algebraic multiplicity of invariant algebraic curves of planar polynomial vector fields.

An application of the extactic polynomial is given by the following result.

Proposition 6. Let $\mathcal{X}$ be the polynomial vector field (1) and let $W$ be a $\mathbb{R}$-vector subspace of $\mathbb{R}[x, y, z]$ of finite dimension $p$, with $p>1$. If $F \in W$ and $\{F=0\}$ is an invariant algebraic surface of $\mathcal{X}$ then $F$ is a factor of $\mathcal{E}_{W}(\mathcal{X})$

If $F \in W$ then it can be chosen as the first element of a basis of $W$. If $\{F=0\}$ is an invariant algebraic surface of $\mathcal{X}$ then (2) holds. By using this fact it is easy to see that $\mathcal{X}^{j}(F)=K_{j} F$, where $K_{1}=K$ and $K_{j}=K\left(K_{j-1}\right)+\mathcal{X}\left(K_{j-1}\right)$ for $j \geq 2$. This and the properties of determinant implies that $F$ is a factor of $\mathcal{E}_{W}(\mathcal{X})$. This is the argument for the proof of Proposition 6.

Our idea is to apply Proposition 6 for studying the invariant meridians and invariant parallels of the polynomial vector field (1) having the algebraic torus $\mathbb{T}^{2}(l, m, n)$ invariant by its flow. Such application is as follows. The maximum number of factors of the form $z-z_{0}$ of the extactic polynomial $\mathcal{E}_{\{1, z\}}(\mathcal{X})$ gives an upper bound for the number of invariant planes $\left\{z-z_{0}=0\right\}$ of $\mathcal{X}$, and this allow to obtain an upper bound for the number of its invariant parallels. Analogously, the maximum number of factors of the form $a x+b y$ of the extactic polynomial $\mathcal{E}_{\{x, y\}}(\mathcal{X})$ allow to obtain an upper bound for the number of invariant meridians of $\mathcal{X}$.

From the definition of the extactic polynomial we get that

$$
\mathcal{E}_{\{1, z\}}(\mathcal{X})=\operatorname{det}\left(\begin{array}{cc}
1 & z \\
\mathcal{X}(1) & \mathcal{X}(z)
\end{array}\right)=\operatorname{det}\left(\begin{array}{ll}
1 & z \\
0 & R
\end{array}\right)=R
$$

and

$$
\mathcal{E}_{\{x, y\}}(\mathcal{X})=\operatorname{det}\left(\begin{array}{cc}
x & y \\
\mathcal{X}(x) & \mathcal{X}(y)
\end{array}\right)=\operatorname{det}\left(\begin{array}{cc}
x & y \\
P & Q
\end{array}\right)=x Q-y P
$$

Remark 1. In general Proposition 6 gives a necessary condition but not a sufficient one. Indeed, in Example 1 we see that the plane $y=2$ is a factor of the extactic polynomial, but it is no a invariant plane of the vector field. However in the case of invariant planes $\{a x+b y=0\}$ and $\left\{z-z_{0}=0\right\}$ the converse statement is true. This is we have the following lemma.

Lemma 7. Let $\mathcal{X}$ be the polynomial vector field (1).

(a) If $z-z_{0}$ is a factor of $\mathcal{E}_{\{1, z\}}(\mathcal{X})$, then $\left\{z-z_{0}=0\right\}$ is an invariant plane of $\mathcal{X}$.

(b) If $a x+b y$ is a factor of $\mathcal{E}_{\{x, y\}}(\mathcal{X})$, then $\{a x+b y=0\}$ is an invariant plane of $\mathcal{X}$. 
Proof. Statement (b) follows immediately, because if $\mathcal{P}=z-z_{0}$ then $P \mathcal{P}_{x}+Q \mathcal{P}_{y}+R \mathcal{P}_{z}=R$. Therefore if $\mathcal{P}$ is a factor of $\mathcal{E}_{\{1, z\}}(\mathcal{X})=R$, then $\mathcal{P}$ satisfies $(2)$.

Let $\mathcal{M}=a x+b y$. If we assume that $\mathcal{E}_{\{x, y\}}(\mathcal{X})=x Q-y P=(a x+b y) T$, where $T$ is a polynomial. Then $x Q-y P=(a x+b y) T$ can be written as $x(Q-a T)=y(b T+P)$. Since $x$ and $y$ have no factors in common, there is a polynomial $S$ such that $Q-a T=y S$ and $b T+P=x S$. Therefore, $P \mathcal{M}_{x}+Q \mathcal{M}_{y}+R \mathcal{M}_{z}=a P+b Q=a(x S-b T)+b(y S+a T)=$ $S(a x+b y)=S \mathcal{M}$. Thus $\mathcal{M}$ satisfies (2). This completes the proof of statement (b).

If $\mathcal{X}$ is polynomial vector field having $\mathbb{T}^{2}(l, m, n)$ invariant by its flow, then Proposition 6 and statement (a) of Lemma 7 transform the study of invariant parallels of $\mathcal{X}$ to the study of factors of the form $z-z_{0}$ of $\mathcal{E}_{\{1, z\}}(\mathcal{X})$. Analogously, Proposition 6 and statement (b) of Lemma 7 transform the study of invariant meridians of $\mathcal{X}$ to the study of factors of the form $a x+b y$ of $\mathcal{E}_{\{x, y\}}(\mathcal{X})$.

\section{Polynomial Vector fields on $\mathbb{T}^{2}(l, m, n)$}

From now on we will consider the polynomials $f=x^{2 l}+y^{2 m}-r^{2}$ and

$$
F=f^{2}+z^{2 n}-1 \text {. }
$$

Hence $\mathbb{T}^{2}(l, m, n)=\{F=0\}$. Thus a polynomial vector field (1) of degree $d$ that satisfies (2) will be called a polynomial vector field of degree $d$ on $\mathbb{T}^{2}(l, m, n)$. Hence $\mathfrak{X}_{d}(l, m, n)$ denotes the set of all polynomial vector field of degree $d$ on $\mathbb{T}^{2}(l, m, n)$.

We recall that $l \geq m$, hence $\operatorname{deg}(f)=2 l$.

Let $\mathcal{X}$ be a polynomial vector field in $\mathfrak{X}_{d}(l, m, n)$, then by using (5) equation (2) can be written as the equation $4 f\left(l x^{2 l-1} P+m y^{2 m-1} Q\right)+2 n z^{2 n-1} R=K\left(f^{2}+z^{2 n}-1\right)$ or equivalently as

$$
z^{2 n-1}(2 n R-z K)=K\left(f^{2}-1\right)-4 f\left(l x^{2 l-1} P+m y^{2 m-1} Q\right) .
$$

In order to obtain general properties on any polynomial vector field on $\mathbb{T}^{2}(l, m, n)$, we note that every polynomial $Y \in \mathbb{R}[x, y, z]$ of degree $d$ can be written as

$$
Y=\sum_{i=0}^{d} Y_{d-i} z^{i}, \quad \text { with } Y_{j} \in \mathbb{R}[x, y] \text { and } \operatorname{deg}\left(Y_{j}\right) \leq j \text { for } j=0,1, \ldots, d
$$

thus if we write the polynomials $P, Q, R$ and $K$ in the previous notation, then the left hand side of (6) becomes

$$
\sum_{i=2 n-1}^{d+2 n-1}\left(2 n R_{d+2 n-1-i}-K_{d+2 n-1-i}\right) z^{i}
$$

and the right side of $(6)$ is

$$
\sum_{i=0}^{d}\left[K_{d-1-i}\left(f^{2}-1\right)-4 f\left(l x^{2 l-1} P_{d-i}+m y^{2 m-1} Q_{d-i}\right)\right] z^{i} .
$$

We must note: first, in (7) the term corresponding to $i=2 n-1$ is $\left(2 n R_{d}-K_{d}\right) z^{2 n-1}$ but $K_{d} \equiv 0$ because $K$ is of degree at most $d-1$; second, in (8) the term corresponding to $i=d$ is $\left[K_{-1}\left(f^{2}-1\right)-4 f\left(l x^{2 l-1} P_{0}+m y^{2 m-1} Q_{0}\right)\right] z^{d}$ but $K_{-1} \equiv 0$ because $K$ is of degree at most $d-1$. Therefore as $(7)$ is equal to (8), then we get the following systems of equations

$$
K_{d-1-i}\left(f^{2}-1\right)-4 f\left(l x^{2 l-1} P_{d-i}+m y^{2 m-1} Q_{d-i}\right) \equiv 0,
$$


for $i=0,1, \ldots, 2 n-2$,

$$
\begin{gathered}
\qquad n R_{d}=K_{d-2 n}\left(f^{2}-1\right)-4 f\left(l x^{2 l-1} P_{d-2 n+1}+m y^{2 m-1} Q_{d-2 n+1}\right), \\
2 n R_{d+2 n-1-i}-K_{d+2 n-1-i}=K_{d-1-i}\left(f^{2}-1\right)-4 f\left(l x^{2 l-1} P_{d-i}+m y^{2 m-1} Q_{d-i}\right), \\
\text { for } i=2 n, 2 n+1, \ldots, d-1, \\
\quad 2 n R_{2 n-1}-K_{2 n-1}=4 f\left(l x^{2 l-1} P_{0}+m y^{2 m-1} Q_{0}\right),
\end{gathered}
$$

and

$$
2 n R_{d+2 n-1-i}-K_{d+2 n-1-i} \equiv 0,
$$

for $i=d+1, d+2, \ldots, d+2 n-1$.

Equation (9) is equivalent to

$$
K_{d-1-i}\left(f^{2}-1\right)=4 f\left(l x^{2 l-1} P_{d-i}+m y^{2 m-1} Q_{d-i}\right),
$$

for $i=0,1, \ldots, 2 n-2$, and since $f$ and $f^{2}-1$ do not have common factors, for every $i=0,1, \ldots, 2 n-2$ there exist $S_{i} \in \mathbb{R}[x, y]$ such that

$$
K_{d-1-i}=4 f S_{i},
$$

and

From (16) we get that

$$
l x^{2 l-1} P_{d-i}+m y^{2 m-1} Q_{d-i}=\left(f^{2}-1\right) S_{i}
$$

$$
\operatorname{deg}\left(S_{i}\right) \leq d-\operatorname{deg}(f)-1-i=d-2 l-1-i,
$$

and that the homogeneous parts of degree less than $2 m-1$ vanish identically.

\section{Proof of Theorem 1}

We will study invariant parallels and invariant meridians through the extactic polynomials $\mathcal{E}_{\{1, z\}}(\mathcal{X})$ and $\mathcal{E}_{\{x, y\}}(\mathcal{X})$ respectively. About invariant parallels we will prove the following result.

Lemma 8. Let $\mathcal{X} \in \mathfrak{X}_{d}(l, m, n)$ having finitely many invariant parallels, then $\mathcal{X}$ has at most $2(d-\min \{2 n, 2 l+1\})$ invariant parallels.

The extactic polynomial $\mathcal{E}_{\{x, y\}}(\mathcal{X})$ can be written as $G_{d}+G_{d-1} z+\cdots+G_{0} z^{d}$, where $G_{i}=x Q_{i}-y P_{i}$ and $\operatorname{deg}\left(G_{i}\right)=i+1$. If $a x+b y$ is a factor of $\mathcal{E}_{\{x, y\}}(\mathcal{X})$ then it is a factor of $G_{i}$ for all $i=0,1, \ldots, d$. Hence we will study the maximum number of factors of the form $a x+b y$ that $G_{i}$ can have. A result in this direction is the following.

Lemma 9. If $\mathcal{X} \in \mathfrak{X}_{d}(l, m, n)$ and $G_{d-i} \not \equiv 0$ for $i=0,1, \ldots, 2 n-2$, then $G_{d-i}$ has at most $d-2 l+1-i$ factors of the form $a x+b y$.

We will assume Lemmas 8 and 9 in order to give the proof of Theorem 1. The proof of these lemmas will be given after the proof of the theorem.

Proof of Theorem 1. Let $\mathcal{X} \in \mathfrak{X}_{d}(l, m, n)$. From Lemma 9 we know that for every $i=$ $0,1, \ldots, 2 n-2$ the polynomial $G_{d-i}$ has at most $d-2 l+1-i$ factors of the form $a x+b y$. The polynomial $G_{d-i}$ has degree $d-i+1$, then for $2 n-1 \leq i \leq d$ the degree of $G_{d-i}$ is at most $d-2 n+2$. Therefore for every $i=0,1, \ldots, d$ the polynomial $G_{d-i}$ has at most $\max \{d-2 l+1, d-2 n+2\}$ factors of the form $a x+b y$.

If $2 n \geq 2 l+1$ then $\min \{2 n, 2 l+1\}=2 l+1$. Hence from Lemma 8 we have that $\mathcal{X}$ has at most $2(d-2 l-1)$ invariant parallels. Moreover if $2 n \geq 2 l+1$ then $\max \{d-2 l+1, d-2 n+2\}=$ 
$d-2 l+1$, so every $G_{d-i}$ has at most $d-2 l+1$ factors of the form $a x+b y$. Therefore by applying Proposition 6 we conclude that $\mathcal{X}$ has at most $2(d-2 l+1)$ invariant meridians. This prove the first part of statement (a).

Now we suppose that $l=m=1$ and consider the polynomial vector field

$$
\mathcal{X}=\left(-y+n\left(f^{2}-1\right) \prod_{i=1}^{d-4}\left(z-z_{i}\right)\right) \frac{\partial}{\partial x}+x \frac{\partial}{\partial y}+2 x z f \prod_{i=1}^{d-4}\left(z-z_{i}\right) \frac{\partial}{\partial z}
$$

of degree $d \geq 5$ and suppose that $0<z_{1}<z_{2}<\cdots<z_{d-4}<1$. $\mathcal{X}$ satisfies (6) taking $K=4 n x f \prod_{i=1}^{d-4}\left(z-z_{i}\right)$, thus $\mathcal{X} \in \mathfrak{X}_{d}(1,1, n)$. Since $\mathcal{E}_{\{1, z\}}(\mathcal{X})=2 x z f \prod_{i=1}^{d-4}\left(z-z_{i}\right)$, from Lemma 7.(a) we obtain that $\mathcal{X}$ has $2(d-4)+2=2(d-3)=2(d-2 l-1)$ invariant parallels.

On the other hand the polynomial vector field

$$
\mathcal{X}=-m y^{2 m-1} T \frac{\partial}{\partial x}+l x^{2 l-1} T \frac{\partial}{\partial y}+0 \frac{\partial}{\partial z}
$$

where $T$ is a polynomial of degree $d-2 l+1$, is of degree $d$ and satisfies (6) taking $K \equiv 0$, thus $\mathcal{X} \in \mathfrak{X}_{d}(l, m, n)$. In addition $\mathcal{E}_{\{x, y\}}(\mathcal{X})=x Q-y P=(n-m) x y z^{2 n-1} T$. Hence, if $T=\prod_{i=1}^{d-2 l+1}\left(a_{i} x+b_{i} y\right)$, with $a_{i}, b_{i} \in \mathbb{R}, a_{i} b_{j} \neq a_{j} b_{i}$ for $i \neq j$, then $\mathcal{E}_{\{x, y\}}(\mathcal{X})$ has $d-2 l+1$ different factors of the form $a x+b y$. Hence from Lemma 7.(b) follows that $\mathcal{X}$ has exactly $2(d-2 l+1)$ invariant meridians. This property is for all $n$, in particular for $2 n \geq 2 l+1$. This complete the proof of statement (a).

If $2 n<2 l+1$ then $\min \{2 n, 2 l+1\}=2 n$. Hence from Lemma 8 we have that $\mathcal{X}$ has at most $2(d-2 n)$ invariant parallels. Moreover if $2 n<2 l+1$ then $\max \{d-2 l+1, d-2 n+2\}=$ $d-2 n+2$, so every $G_{d-i}$ has at most $d-2 n+2$ factors of the form $a x+b y$. Therefore by applying Proposition 6 we conclude that $\mathcal{X}$ has at most $2(d-2 n+2)$ invariant meridians. This prove the first part of statement (b). Now suppose $l=n$, thus $2 n<2 l+1$. Let $T$ be a polynomial of degree $d-2 n$ and consider the polynomial vector field

$$
\mathcal{X}=m x z^{2 n-1} T \frac{\partial}{\partial x}+n z^{2 n-1} T \frac{\partial}{\partial y}+2 m\left(z^{2 n}-1-r^{2} f\right) T \frac{\partial}{\partial z},
$$

which satisfies (6) taking $K=4 m n z^{2 n-1} T$, thus $\mathcal{X} \in \mathfrak{X}_{d}(n, m, n)$.

If $T=\prod_{i=1}^{d-2 n}\left(z-z_{i}\right)$ then $\mathcal{E}_{\{1, z\}}(\mathcal{X})=2 m\left(z^{2 n}-1-r^{2} f\right) T$ has $d-2 n$ factors of the form $z-z_{0}$ because $z^{2 n}-1-r^{2} f$ is irreducible. Therefore if for $i=1,2, \ldots, d-2 n,\left|z_{i}\right|<1$ and $z_{i} \neq z_{j}$ for $i \neq j$, then from Lemma 7 . (a) we get that $\mathcal{X}$ has $2(d-2 n)$ invariant parallels. On the other hand, if $T=\prod_{i=1}^{d-2 n}\left(a_{i} x+b_{i} y\right)$, with $a_{i}, b_{i} \in \mathbb{R} \backslash\{0\}, a_{i} b_{j} \neq a_{j} b_{i}$ for $i \neq j$, then

$$
\mathcal{E}_{\{x, y\}}(\mathcal{X})=x Q-y P=(n-m) x y z^{2 n-1} T
$$

has $d-2 n+2$ different factors of the form $a x+b y$. Hence from Lemma 7.(b) follows that $\mathcal{X}$ has exactly $2(d-2 n+2)$ invariant meridians. This complete the proof of statement $(\mathrm{b})$.

Let $\mathcal{X} \in \mathfrak{X}_{d}(l, m, n)$. From statement (b) of Lemma 7 the number of invariant parallels of $\mathcal{X}$ is bounded by two times the maximum number of factors of the form $z-z_{0}\left(z_{0} \in[-1,1]\right)$ of the extactic polynomial $\mathcal{E}_{\{1, z\}}(\mathcal{X})=R$. We note that $z-z_{0}$ is a factor of $R$ if and only if $z-z_{0}$ is a factor of $R\left(x_{0}, y_{0}, z\right)$ for a point $\left(x_{0}, y_{0}\right) \in \mathbb{R}^{2}$. Then we will prove Lemma 8 by finding the maximum number of factors of the form $z-z_{0}$ of $R\left(x_{0}, y_{0}, z\right)$, where $\left(x_{0}, y_{0}\right)$ is a particular point. 
Proof of Lemma 8. As (6) holds for all $(x, y, z) \in \mathbb{R}^{3}$, in particular it holds for the points $(0,0, z)$. Thus in such case $(6)$ becomes

$$
z^{2 n-1}(2 n R(0,0, z)-z K(0,0, z))=K(0,0, z)\left(r^{4}-1\right) .
$$

Then there exists a polynomial $T$ of degree at most $d-2 n$ such that $K(0,0, z)=z^{2 n-1} T$ and $2 n R(0,0, z)-z K(0,0, z)=\left(r^{4}-1\right) T$. Hence $2 n R(0,0, z)=\left(r^{4}+z^{2 n}-1\right) T$. We can consider two cases: either $R(0,0, z) \not \equiv 0$ or $R(0,0, z) \equiv 0$.

Case 1 . Suppose $R(0,0, z) \not \equiv 0$. As $r^{4}+z^{2 n}-1$ is not divisible by polynomials of the form $z-z_{0}$, then the maximum number of factors of the form $z-z_{0}$ of $R(0,0, z)$ is $\operatorname{deg}(T)=d-2 n$. Therefore $\mathcal{E}_{\{1, z\}}(\mathcal{X})$ has at most $d-2 n$ factors of the form $z-z_{0}$. Hence $\mathcal{X}$ has at most $2(d-2 n)$ invariant parallels.

Case 2. Suppose $R(0,0, z) \equiv 0$. We follow the same ideas as in previous case, i.e. as (6) holds for all $(x, y, z) \in \mathbb{R}^{3}$, in particular it holds for the points $\left(x_{0}, y_{0}, z\right)$ with $f\left(x_{0}, y_{0}\right)=0$. Thus in such case $(6)$ becomes $z^{2 n-1}\left(2 n R\left(x_{0}, y_{0}, z\right)-z K\left(x_{0}, y_{0}, z\right)\right)=K\left(x_{0}, y_{0}, z\right)$ which can be written as

$$
2 n z^{2 n-1} R\left(x_{0}, y_{0}, z\right)=K\left(x_{0}, y_{0}, z\right)\left(z^{2 n}-1\right) .
$$

Since $z^{2 n-1}$ and $z^{2 n}-1$ do not have factors in common, there exists a polynomial $T$ such that $R\left(x_{0}, y_{0}, z\right)=\left(z^{2 n}-1\right) T$ and $K\left(x_{0}, y_{0}, z\right)=2 n z^{2 n-1} T$. From this last condition on $T$ we obtain that the degree of $T$ is at most $\operatorname{deg}\left(K\left(x_{0}, y_{0}, z\right)\right)-2 n+1$. Since in this case $K(0,0, z) \equiv 0, K_{0}=0$. Hence $\operatorname{deg}\left(K\left(x_{0}, y_{0}, z\right)\right) \leq d-2$ for any point $\left(x_{0}, y_{0}\right) \in \mathbb{R}^{2}$. Therefore $\operatorname{deg}(T) \leq d-2 n-1$ Again we have two subcases: either $R\left(x_{0}, y_{0}, z\right) \not \equiv 0$ or $R\left(x_{0}, y_{0}, z\right) \equiv 0$.

Subcase 2.1. Suppose $R\left(x_{0}, y_{0}, z\right) \not \equiv 0$ for a point $\left(x_{0}, y_{0}\right)$ such that $f\left(x_{0}, y_{0}\right)=0$. We have proved that $R\left(x_{0}, y_{0}, z\right)=\left(z^{2 n}-1\right) T$ and $\operatorname{deg}(T) \leq d-2 n-1$. Therefore $R\left(x_{0}, y_{0}, z\right)$ has the two factors $z-1$ and $z+1$, which are the unique real factors of $z^{2 n}-1$, and has at most $d-2 n-1$ factors of the form $z-z_{0}$, with $z_{0} \in(-1,1)$. So $\mathcal{E}_{\{1, z\}}(\mathcal{X})$ has the two factors $z-1$ and $z+1$, and has at most $d-2 n-1$ factors of the form $z-z_{0}$, with $-1<z_{0}<1$. Hence $\mathcal{X}$ has at most $2(d-2 n-1)+2=2(d-2 n)$ invariant parallels.

Subcase 2.2. Suppose $R\left(x_{0}, y_{0}, z\right) \equiv 0$ for every point $\left(x_{0}, y_{0}\right)$ of the algebraic curve $\{f=0\}$. Then $f$ is a factor of $R(x, y, z)$, i.e. $R(x, y, z)=f h(x, y, z)$, in addition $R_{0}=0$. Hence the maximum power of $z$ in $R(x, y, z)$ is at most $d-1$. Therefore the maximum power of $z$ in $h(x, y, z)$ is at most $d-1-\operatorname{deg}(f)=d-2 l-1$. So $\mathcal{E}_{\{1, z\}}(\mathcal{X})$ has at most $d-2 l-1$ factors of the form $z-z_{0}$. Hence $\mathcal{X}$ has at most $2(d-2 l-1)$ invariant parallels.

Proof of Lemma 9. We have that $G_{d-i}=x Q_{d-i}-y P_{d-i}$. For $i=0,1, \ldots, 2 n-2$ the equation (16) can be written as

$$
l x^{2 l-1} P_{d-i}+m y^{2 m-1} Q_{d-i}-\left(x^{2 l}+y^{2 m}\right)\left(x^{2 l}+y^{2 m}-2 r^{2}\right) S_{i}=\left(r^{4}-1\right) S_{i} .
$$

We can write the polynomial $P_{d-i}$ as $P_{d-i}=\bar{P}_{d-i}+\widetilde{P}_{d-i}$, where $\bar{P}_{d-i}$ is the sum of the homogeneous parts of degree at most $\operatorname{deg}\left(S_{i}\right)-2 l+1$ of $P_{d-i}$, and $\widetilde{P}_{d-i}=P_{d-i}-\bar{P}_{d-i}$. Analogously, we write the polynomial $Q_{d-i}$ as $Q_{d-i}=\bar{Q}_{d-i}+\widetilde{Q}_{d-i}$, where $\bar{Q}_{d-i}$ is the sum of the homogeneous parts of degree at most $\operatorname{deg}\left(S_{i}\right)-2 m+1$ of $Q_{d-i}$, and $\widetilde{Q}_{d-i}=Q_{d-i}-\bar{Q}_{d-i}$. Then from previous equation we have that

$$
l x^{2 l-1} \bar{P}_{d-i}+m y^{2 m-1} \bar{Q}_{d-i}=\left(r^{4}-1\right) S_{i}
$$

and

$$
l x^{2 l-1} \widetilde{P}_{d-i}+m y^{2 m-1} \widetilde{Q}_{d-i}-\left(x^{2 l}+y^{2 m}\right)\left(x^{2 l}+y^{2 m}-2 r^{2}\right) S_{i} \equiv 0 .
$$


By reordering terms the last equation is equivalent to the following

$$
x^{2 l-1}\left(l \widetilde{P}_{d-i}-x\left(f-r^{2}\right) S_{i}\right)=-y^{2 m-1}\left(m \widetilde{Q}_{d-i}-y\left(f-r^{2}\right) S_{i}\right) .
$$

Since $x^{2 l-1}$ and $y^{2 m-1}$ do not have common factors, there exist a polynomial $T_{i}$ such that $l \widetilde{P}_{d-i}-x\left(f-r^{2}\right) S_{i}=-y^{2 m-1} T_{i}$ and $m \widetilde{Q}_{d-i}-y\left(f-r^{2}\right) S_{i}=x^{2 l-1} T_{i}$, whence

$$
\widetilde{P}_{d-i}=\left(-y^{2 m-1} T_{i}+x\left(f-r^{2}\right) S_{i}\right) / l \quad \text { and } \quad \widetilde{Q}_{d-i}=\left(x^{2 l-1} T_{i}+y\left(f-r^{2}\right) S_{i}\right) / m .
$$

Therefore

$$
G_{d-i}=x \bar{Q}_{d-i}-y \bar{P}_{d-i}+\frac{l x^{2 l}+m y^{2 m}}{l m} T_{i}+\frac{(l-m) x y\left(f-r^{2}\right)}{l m} S_{i} .
$$

Now, as $\operatorname{deg}\left(\bar{P}_{d-i}\right) \leq \operatorname{deg}\left(S_{i}\right)-2 l+1, \operatorname{deg}\left(\bar{Q}_{d-i}\right) \leq \operatorname{deg}\left(S_{i}\right)-2 m+1$, and $\operatorname{deg}\left(S_{i}\right) \leq$ $d-2 l-1-i$, then $\operatorname{deg}\left(\bar{P}_{d-i}\right) \leq d-2 l-2 l-i$ and $\operatorname{deg}\left(\bar{Q}_{d-i}\right) \leq d-2 l-2 m-i$. Hence $\operatorname{deg}\left(x \bar{Q}_{d-i}-y \bar{P}_{d-i}\right) \leq \max \{d-2 l-2 l-i+1, d-2 l-2 m-i+1\}=d-2 l-2 m+1-i$.

If $x \bar{Q}_{d-i}-y \bar{P}_{d-i} \not \equiv 0$, then $G_{d-i}$ has at most $d-2 l-2 m+1-i$ factors of the form $a x+b y$, and as $d-2 l-2 m+1-i<d-2 l+1-i$ then assertion follows.

On the other hand, if $x \bar{Q}_{d-i}-y \bar{P}_{d-i} \equiv 0$ then there exists a polynomial $V_{i}$ such that $\bar{Q}_{d-i}=y V_{i}$ and $\bar{P}_{d-i}=x V_{i}$, moreover from these conditions it follows that $\operatorname{deg}\left(V_{i}\right) \leq$ $\operatorname{deg}\left(\bar{P}_{d-i}\right)-1 \leq d-4 l-1-i$. Hence from (18) we get that $S_{i}=\left(l x^{2 l}+m y^{2 m}\right) V_{i} /\left(r^{4}-1\right)$. Therefore (19) reduces to

$$
G_{d-i}=\frac{l x^{2 l}+m y^{2 m}}{l m\left(r^{4}-1\right)}\left[\left(r^{4}-1\right) T_{i}+(l-m) x y\left(f-r^{2}\right) V_{i}\right] .
$$

If $V_{i} \not \equiv 0$ then $G_{d-i}$ has at most $d-4 l+1-i$ factors of the form $a x+b y \operatorname{because} \operatorname{deg}\left(x y V_{i}\right) \leq$ $d-4 l+1-i$ and $l x^{2 l}+m y^{2 m}$ is irreducible, moreover as $d-4 l+1-i<d-2 l+1-i$ then assertion follows.

Finally, $V_{i} \equiv 0$ then $G_{d-i}=\left(l x^{2 l}+m y^{2 m}\right) T_{i} /(l m)$ has at most $d-2 l+1-i$ factors of the form $a x+b y$ because $\operatorname{deg}\left(T_{i}\right) \leq d-2 l+1-i$ and $l x^{2 l}+m y^{2 m}$ is irreducible.

\section{Proof of Theorem 2}

We will prove Theorem 2 by assuming the following result

Lemma 10. If $d<2 l-1$ then every element of $\mathfrak{X}_{d}(l, m, n)$ has the form

$$
\mathcal{X}=m n x y^{2 m-1} z^{2 n-1} T \frac{\partial}{\partial x}+\ln \left(r^{2}-y^{2 m}\right) z^{2 n-1} T \frac{\partial}{\partial y}+2 \ln y^{2 m-1}\left(z^{2 n}-1\right) T \frac{\partial}{\partial z},
$$

where $T$ is a polynomial of degree at most $d-2(m+n)+1$.

We will prove this lemma after the proof of the theorem.

Proof of Theorem 2. Let $\mathcal{X}$ be a polynomial vector field in $\mathfrak{X}_{d}(l, m, n)$.

Proof of statement (a). If $d<2(m+n)-1$ then from Lemma $10, T \equiv 0$, hence $\mathcal{X} \equiv 0$.

Proof of statement (b). From Lemma 10 we get that $\mathcal{X} \neq 0$ if and only if $T \neq 0$. Hence the extactic polynomials

$$
\mathcal{E}_{\{1, z\}}(\mathcal{X})=R=2 l m y^{2 m-1}\left(z^{2 n}-1\right) T
$$

and

$$
\mathcal{E}_{\{x, y\}}(\mathcal{X})=x Q-y P=-x z^{2 n-1}\left(l r^{2}-(l-m) y^{2 m}\right) T
$$


do not vanish identically. Therefore $\mathcal{X}$ has finitely many invariant parallels and invariant meridians. In addition, $\mathcal{E}_{\{1, z\}}(\mathcal{X})$ has at most $(d-2(m+n)+1)$ factors of the form $z-z_{0}$ with $z_{0} \neq \pm 1$ and has the two factors $z-1$ and $z+1$, which implies that $\mathcal{X}$ has at least two and at most $2(d-2(m+n)+2)$ invariant parallels. On the other hand $\mathcal{E}_{\{x, y\}}(\mathcal{X})$ has at most $d-2(m+n)+1$ factors of the form $a x+b y$, with $b \neq 0$ and has the factor $x$, which implies that $\mathcal{X}$ has at least two and at most $2(d-2(m+n)+2)$ invariant meridians. Therefore the sum of invariant parallels and meridians is at least four and at most $2(\operatorname{deg}(T))+4=2(d-2(m+n)+3)$.

Proof of statement (c). As $x=0, z=1$, and $z=-1$ are always invariant planes of $\mathcal{X}$, then always $\mathcal{X}$ has two invariant meridians $\mathcal{M}_{1} \cup \mathcal{M}_{2}=\{x=0\} \cap \mathbb{T}^{2}(l, m, n)$ and two invariant parallels $\mathcal{P}_{1}=\{z-1=0\} \cap \mathbb{T}^{2}(l, m, n)$ and $\mathcal{P}_{2}=\{z+1=0\} \cap \mathbb{T}^{2}(l, m, n)$. This implies that any invariant parallel intersects $\mathcal{M}_{1}$ and $\mathcal{M}_{2}$. Therefore the invariant parallel cannot be a limit cycle. Analogously, any invariant meridian intersects to $\mathcal{P}_{1}$ and $\mathcal{P}_{2}$.

Finally, statement (d) follows from the property that $T$ is an arbitrary polynomial of degree $d-2(m+n)+1$ and that $\mathcal{P}_{1}$ and $\mathcal{P}_{2}$, and $\mathcal{M}_{1}$ and $\mathcal{M}_{2}$, are always invariant parallels and invariant meridians respectively.

Proof of Lemma 10. Let $\mathcal{X} \in \mathfrak{X}_{d}(l, m, n)$ be a polynomial vector field (1) of degree $d$ on $\mathbb{T}^{2}(l, m, n)$. We are assuming that $d<2 l-1$ then from $(17) \operatorname{deg}\left(S_{i}\right)<-i \leq 0$, thus $S_{i} \equiv 0$. Hence from (15) we get that $K_{d-1-i} \equiv 0$ for $i=0,1, \ldots, 2 n-2$, and from (16) we get that $l x^{2 l-1} P_{d-i}=-m y^{2 m-1} Q_{d-i}$. Therefore there exists a polynomial $T_{i} \in \mathbb{R}[x, y]$ such that $P_{d-i}=-m y^{2 m-1} T_{i}$ and $Q_{d-i}=l x^{2 l-1} T_{i}$. Now, for $i=0,1, \ldots, 2 n-2$ the polynomial $Q_{d-i}$ is of degree at most $d-i$ and as $d-i<2 l-1-i \leq 2 l-1$, then $T_{i} \equiv 0$, which implies that $P_{d-i} \equiv Q_{d-i} \equiv 0$.

Form the previous paragraph we have that

$$
P=z^{2 n-1} \widetilde{P}, \quad Q=z^{2 n-1} \widetilde{Q} \text { and } K=z^{2 n-1} \widetilde{K} .
$$

Then (6) reduces to $2 n R-z^{2 n} \widetilde{K}=\widetilde{K}\left(f^{2}-1\right)-4 f\left(l x^{2 l-1} \widetilde{P}+m y^{2 m-1} \widetilde{Q}\right)$, which can be written as

$$
2 n R-\widetilde{K}\left(z^{2 n}-1\right)=f\left(x^{2 l-1}(4 l \widetilde{P}-x \widetilde{K})+y^{2 m-1}(4 m \widetilde{Q}-y \widetilde{K})\right)+r^{2} f \widetilde{K} .
$$

If $4 l \widetilde{P}-x \widetilde{K}$ does not vanish identically then the right hand side of (21) is a polynomial of degree at least $4 l-1>d$, while the left hand side has degree at most $d$. Hence $4 l \widetilde{P}-x \widetilde{K} \equiv 0$, thus there exists $\bar{K} \in \mathbb{R}[x, y]$ such that

$$
\widetilde{K}=4 \ln \bar{K}, \quad \widetilde{P}=n x \bar{K},
$$

and equation (21) reduces to

$$
2 n R-4 \ln \bar{K}\left(z^{2 n}-1\right)=f\left(4 \ln \bar{K}\left(r^{2}-y^{2 m}\right)+4 m y^{2 m-1} \widetilde{Q}\right) .
$$

If $4 \ln \bar{K}\left(r^{2}-y^{2 m}\right)+4 m y^{2 m-1} \widetilde{Q}$ does not vanish identically then the left hand side of the previous equation is a polynomial of degree at least $2 l>d$, while the right hand side has degree at most $d$. Hence $4 \ln \bar{K}\left(r^{2}-y^{2 m}\right)=-4 m y^{2 m-1} \widetilde{Q}$. Since $y^{2 m-1}$ and $r^{2}-y^{2 m}$ does not have common factors, there exists $T \in \mathbb{R}[x, y]$ such that

$$
\bar{K}=m y^{2 m-1} T, \quad \widetilde{Q}=\ln \left(r^{2}-y^{2 m}\right) T \quad \text { and } \quad 2 n R=-4 \ln \bar{K}\left(z^{2 n}-1\right) .
$$

By using (20), (22) and (23) we prove that

$$
P=m n x y^{2 m-1} z^{2 n-1} T, Q=\ln \left(r^{2}-y^{2 m}\right) z^{2 n-1} T \quad \text { and } \quad R=2 l m y^{2 m-1}\left(z^{2 n}-1\right) T .
$$


It is clear that $\operatorname{deg}(T) \leq d-2(n+m)+1$.

\section{Proof of Theorems 3 And 4}

In this section we are assuming that $l=m=n$. This is $f=x^{2 n}+y^{2 n}-r^{2}$ and $F=f^{2}+z^{2 n}-1$.

Proof of Theorem 3. Let $\mathcal{X}$ be a polynomial vector field in $\mathfrak{X}_{d}(n, n, n)$.

Proof of statement (a). If $d<2 n-1$ then from statement (a.1) of Theorem $2, \mathcal{X} \equiv 0$. Therefore, if $\mathcal{X}$ has finitely many invariant meridians or invariant parallels, then $d \geq 2 n-1$.

Statement (b) follows from statement (b) of Theorem 1.

Proof of statement (c). For for $i=0,1, \ldots, d$ the polynomial $G_{d-i}$ has degree $d-i+1$, then for $i=2 n, 2 n+1, \ldots, d$ the degree of $G_{d-i}$ is at most $d-2 n+1$. Moreover from Lemma 9 we know that for $i=0,1, \ldots, 2 n-2$ the polynomial $G_{d-i}$ has at most $d-2 n+1-i \leq d-2 n+1$ factors of the form $a x+b y$. Therefore to complete the proof we only need to prove that $G_{d-2 n+1}=x Q_{d-2 n+1}-y P_{d-2 n+1}$ has at most $d-2 n+1$ factors of the form $a x+b y$. Next we will prove this fact.

From (10) we know that $P_{d-2 n+1}$ and $Q_{d-2 n+1}$ satisfy the equation

$$
2 n R_{d}=K_{d-2 n}\left(f^{2}-1\right)-4 f\left(l x^{2 l-1} P_{d-2 n+1}+m y^{2 m-1} Q_{d-2 n+1}\right),
$$

which can be written as

$$
\begin{aligned}
2 n R_{d}= & f\left[x^{2 n-1}\left(x K_{d-2 n}-4 n P_{d-2 n+1}\right)+y^{2 n-1}\left(y K_{d-2 n}-4 n Q_{d-2 n+1}\right)\right] \\
& -K_{d-2 n}\left(1+r^{2} f\right) .
\end{aligned}
$$

Let $\widetilde{P}_{d-2 n+1}$ and $\widetilde{Q}_{d-2 n+1}$ be the sum of the homogeneous parts of degree at least $d-4 n+2$ of $P_{d-2 n+1}$ and $Q_{d-2 n+1}$ respectively. Let $\widetilde{K}_{d-2 n}$ be the sum of the homogeneous parts of degree at least $d-4 n+1$ of $K_{d-2 n}$. Then from previous equation we have that if $x^{2 n-1}\left(x \widetilde{K}_{d-2 n}-4 n \widetilde{P}_{d-2 n+1}\right)+y^{2 n-1}\left(y \widetilde{K}_{d-2 n}-4 n \widetilde{Q}_{d-2 n+1}\right)$ does not vanish identically, then the degree of the right hand side is at least $d+1>d$, while the left hand side is of degree $d$. Therefore

$$
x^{2 n-1}\left(x \widetilde{K}_{d-2 n}-4 n \widetilde{P}_{d-2 n+1}\right)=-y^{2 n-1}\left(y \widetilde{K}_{d-2 n}-4 n \widetilde{Q}_{d-2 n+1}\right) .
$$

Since $x^{2 n-1}$ and $y^{2 n-1}$ do not have common factor, there exists $T \in \mathbb{R}[x, y]$ such that

$$
x \widetilde{K}_{d-2 n}-4 n \widetilde{P}_{d-2 n+1}=y^{2 n-1} T \text { and } y \widetilde{K}_{d-2 n}-4 n \widetilde{Q}_{d-2 n+1}=-x^{2 n-1} T,
$$

or equivalently

$$
\widetilde{P}_{d-2 n+1}=-y^{2 n-1} T+x \widetilde{K}_{d-2 n} \quad \text { and } \quad \widetilde{Q}_{d-2 n+1}=x^{2 n-1} T+y \widetilde{K}_{d-2 n} .
$$

Thus $x \widetilde{Q}_{d-2 n+1}-y \widetilde{P}_{d-2 n+1}=\left(x^{2 n}+y^{2 n}\right) T$ and we have that $\operatorname{deg}(T) \leq d-4 n+2$. Therefore

$$
G_{d-2 n+1}=x Q_{d-2 n+1}-y P_{d-2 n+1}=\bar{G}+\left(x^{2 n}+y^{2 n}\right) T,
$$

where $\bar{G}=x\left(Q_{d-2 n+1}-\widetilde{Q}_{d-2 n+1}\right)-y\left(P_{d-2 n+1}-\widetilde{P}_{d-2 n+1}\right)$ is of degree at most $d-4 n+1$. As a result $G_{d-2 n+1}$ has at most $d-4 n+2$ factors of the form $a x+b y$.

Proof of Theorem 4. Let $d$ and $n$ be positive integers such that $d \geq 2 n-1$. For every $k \in\{0,1, \ldots, d-2 n+1\}$ we define the nonnegative number $\alpha_{k}=d-2 n-k+1$, the 
polynomial $H_{1}^{k}=\prod_{i=1}^{k} a_{i} x+b_{i} y$, where $a_{i}, b_{i} \in \mathbb{R}, a_{i} b_{j} \neq a_{j} b_{i}$ for $i \neq j$; if $k=0, H_{1}^{0}=1$. Consider the polynomial vector field

$$
\mathcal{X}=P \frac{\partial}{\partial x}+Q \frac{\partial}{\partial y}+R \frac{\partial}{\partial z}
$$

where $P=-y^{2 n-1}\left(z-z_{0}\right)^{\alpha_{k}} H_{1}^{k}+x z^{2 n-1} H_{2}, Q=x^{2 n-1}\left(z-z_{0}\right)^{\alpha_{k}} H_{1}^{k}+y z^{2 n-1} H_{2}, R=$ $2\left(z^{2 n}-1-r^{2} f\right) H_{2},\left|z_{0}\right|>1, H_{2}=\prod_{i=1}^{d-2 n} z-z_{i}, z_{i} \in \mathbb{R}, H_{2}=1$ if $d=2 n$, and $H_{2}=0$ if $d=2 n-1$.

The polynomial vector field $\mathcal{X}$ is of degree $d$ and satisfies (6) taking $K=4 n z^{2 n-1} H_{2}$, then $\mathcal{X} \in \mathfrak{X}_{d}(n, n, n)$. In addition we have that

$$
\mathcal{E}_{\{x, y\}}(\mathcal{X})=x Q-y P=\left(x^{2 n}+y^{2 n}\right)\left(z-z_{0}\right)^{\alpha_{k}} H_{1}^{k},
$$

and

$$
\mathcal{E}_{\{1, z\}}(\mathcal{X})=R=2\left(z^{2 n}-1-r^{2} f\right) H_{2} .
$$

So $\mathcal{X}$ has $k$ invariant planes $a_{i} x+b_{i} y$, hence $\mathcal{X}$ has $2 k$ invariant meridians.

On the other hand for every $\nu \in\{0,1,2, \ldots, 2(d-2 n)\}$ there is $\mu \in\{0,1,2, \ldots, d-2 n\}$ and $\tau \in\{0,1\}$ such that $\nu=2 \mu-\tau$. If $\tau=1$ then we consider $H_{2}$ in such a way that $-1=z_{1}<z_{2}<\cdots<z_{\mu}<1$ and $\left|z_{i}\right|>1$ for $\mu<i<d-2 n$, otherwise $-1<z_{1}<z_{2}<$ $\cdots<z_{\mu}<1$ and $\left|z_{i}\right|>1$ for $\mu<i<d-2 n$. Thus, we obtain that $\mathcal{X}$ has exactly $\nu=2 \mu-\tau$ invariant parallels.

Now we consider $\mathcal{X}$ with $H_{2}=1$. If $\left(x^{*}, y^{*}, z^{*}\right) \in \mathbb{T}^{2}(n, n, n)$ is a singular point of $\mathcal{X}$, then $\left(x^{*}, y^{*}\right) \neq(0,0)$, thus $\left(x^{*}\right)^{2 n}+\left(y^{*}\right)^{2 n}>0$. Since $z^{*}-z_{0} \neq 0$ and $0=x^{*} Q\left(x^{*}, y^{*}, z^{*}\right)-$ $y^{*} P\left(x^{*}, y^{*}, z^{*}\right)=\left(\left(x^{*}\right)^{2 n}+\left(y^{*}\right)^{2 n}\right)\left(z^{*}-z_{0}\right)^{\alpha_{k}} H_{1}^{k}\left(x^{*}, y^{*}\right), H_{1}^{k}\left(x^{*}, y^{*}\right)=0$. Hence using the expressions of $P$ and $Q$ we obtain that $x^{*}\left(z^{*}\right)^{2 n-1}=0$ and $y^{*}\left(z^{*}\right)^{2 n-1}=0$, hence $z^{*}=0$. Now as $\left(x^{*}, y^{*}, z^{*}\right)=\left(x^{*}, y^{*}, 0\right) \in \mathbb{T}^{2}(n, n, n)$ then $f\left(x^{*}, y^{*}\right)= \pm 1$, thus $R\left(x^{*}, y^{*}, 0\right)=$ $-2\left(1 \pm r^{2}\right) \neq 0$. In conclusion $\mathcal{X}$ has no singular points on $\mathbb{T}^{2}(n, n, n)$, therefore the $2 k$ invariant meridians of $X$ are periodic orbits. In addition the differential system associated to $\mathcal{X}$ in cylindrical coordinates satisfies that

$$
\dot{\theta}=(z-z 0)^{\alpha_{k}}\left(r^{2 n}\left(\cos ^{2 n} \theta+\sin ^{2 n} \theta\right)\right) H_{1}^{k} .
$$

Since $\left|z_{0}\right|>1$, between two consecutive invariant meridians the

$$
\operatorname{sign}(\dot{\theta})=\operatorname{sign}\left(H_{1}^{k}(r \cos \theta, r \sin \theta) \in\{-1,1\},\right.
$$

and these signs change when we cross one meridian. So the periodic orbits on the invariant meridians are stable or unstable (algebraic) limit cycles alternately.

Following the previous idea we can prove that if we consider $\mathcal{X}$ with $H_{1}^{0}$ the $\mathcal{X}$ has $\nu$ invariant parallels which are (algebraic) limit cycles.

\section{Proof of Theorem 5}

The idea to prove Theorem 5 is as follows. From the Case 1 of proof of Lemma 8 we know that if $R(0,0, z)$ does not vanish identically then $\mathcal{X}$ has at most $2(d-2 n)$ invariant parallels. Then we will prove the following lemma.

Lemma 11. If $l=m$ and $d \geq 2 n \geq 2 l$, or if $l>m$ and $d \geq 2 n \geq 2(l+m)$, then the subset $\mathcal{R}_{d} \subset \mathfrak{X}_{d}(l, m, n)$ of polynomial vector fields such that $R(0,0, z) \not \equiv 0$ is generic.

On the other hand, if $\mathcal{X} \in \mathfrak{X}_{d}(l, m, n)$ and $G_{d} \not \equiv 0$ then from Lemma 9 we know that $G_{d}$ has at most $d-2 l+1$ factors of the form $a x+b y$ which implies that $\mathcal{X}$ has at most $2(d-2 l+1)$ invariant meridians. Then we will prove the following lemma. 
Lemma 12. If $d \geq 2 l-1$ then the subset $\mathcal{G}_{d} \subset \mathfrak{X}_{d}(l, m, n)$ of polynomial vector fields such that $G_{d}=x Q_{d}-y P_{d} \not \equiv 0$ is generic.

As a result we will obtain that $\mathcal{R}_{d} \cap \mathcal{G}_{d}$ will be the desired generic subset for the proof of the first two statements of Theorem 5. For proving the next two statements we will construct a polynomial vector field $\mathcal{X}$ having the desired properties. That construction is as follows: Let $d, l, m$ and $n$ be positive integers such that either $d \geq 2 n \geq 2 l$ if $l=m$, or $d \geq 2 n \geq 2(l+m)$ if $l>m$. For every $k \in\{0,1, \ldots, d-2 l+1\}$ we define the nonnegative number $\alpha_{k}=d-2 l-k+1$ and the polynomial $H_{1}^{k}=\prod_{i=1}^{k} a_{i} x+b_{i} y$ of degree $k$, where $a_{i}, b_{i} \in \mathbb{R}, a_{i} b_{j} \neq a_{j} b_{i}$ for $i \neq j$; if $k=0, H_{1}^{0}=c t e$. Consider the polynomial vector field

$$
\mathcal{X}=P \frac{\partial}{\partial x}+Q \frac{\partial}{\partial y}+R \frac{\partial}{\partial z}
$$

where $P=-m y^{2 m-1}\left(z-z_{0}\right)^{\alpha_{k}} H_{1}^{k}+n x z^{2 n-1} H_{2}, Q=l x^{2 l-1}\left(z-z_{0}\right)^{\alpha_{k}} H_{1}^{k}+n y z^{2 n-1} H_{2}$, $R=2\left((l-m) y^{2 m} f+l\left(z^{2 n}-1-r^{2} f\right)\right) H_{2},\left|z_{0}\right|>1, H_{2}=\prod_{i=1}^{d-2 n} z-z_{i}, z_{i} \in \mathbb{R}$, and $H_{2}=$ cte if $d-2 n=0$.

Lemma 13. The polynomial vector field $\mathcal{X}$ given by (24) satisfies that $\mathcal{X} \in \mathcal{R}_{d} \cap \mathcal{G}_{d}$.

Next we are going to give the proof of Theorem 5 by assuming the three previous lemmas whose proofs will be given after the proof of the theorem.

Proof of Theorem 5. In both cases: if $l=m$ and $d \geq 2 n \geq 2 l$, or if $l>m$ and $d \geq 2 n \geq$ $2(l+m)$ we know from Lemma 11 that $\mathcal{R}_{d}$ is a generic subset of $\mathfrak{X}_{d}(l, m, n)$. Moreover as $2(l+m)>2 l>2 l-1$ and $d \geq 2 l$ or $d \geq 2(l+m)$, then from Lemma 12 follows that $\mathcal{G}_{d}$ is also a generic subset of $\mathfrak{X}_{d}(l, m, n)$. Therefore $\mathcal{R}_{d} \cap \mathcal{G}_{d}$ is a generic subset of $\mathfrak{X}_{d}(l, m, n)$ and for every $\mathcal{X} \in \mathcal{R}_{d} \cap \mathcal{G}_{d}$ the maximum number of invariant parallels is $2(d-2 n)$, and the maximum number of invariant meridians is $2(d-2 l+1)$. This prove statements (a) and (b).

Now, let $\mathcal{X}$ be the vector field given by (24). From Lemma 13 we know that $\mathcal{X} \in \mathcal{R}_{d} \cap \mathcal{G}_{d}$.

For every $\nu \in\{0,1,2, \ldots, 2(d-2 n)\}$ there is $\mu \in\{0,1,2, \ldots, d-2 n\}$ and $\tau \in\{0,1\}$ such that $\nu=2 \mu-\tau$. If $\tau=1$ then in $\mathcal{X}$ we consider $H_{2}$ in such a way that $-1=z_{1}<z_{2}<$ $\cdots<z_{\mu}<1$ and $\left|z_{i}\right|>1$ for $\mu<i<d-2 n$, otherwise $-1<z_{1}<z_{2}<\cdots<z_{\mu}<1$ and and $\left|z_{i}\right|>1$ for $\mu<i<d-2 n$. Hence as

$$
\mathcal{E}_{\{1, z\}}(\mathcal{X})=R=2\left((l-m) y^{2 m} f+l\left(z^{2 n}-1-r^{2} f\right)\right) H_{2}
$$

and $(l-m) y^{2 m} f+l\left(z^{2 n}-1-r^{2} f\right)$ does not have any factor of the form $z-z_{0}$, then from Lemma 7.(a) it follows that $\mathcal{X}$ has exactly $\nu=2 \mu-\tau$ invariant parallels.

Also we have that

$$
\mathcal{E}_{\{x, y\}}(\mathcal{X})=x Q-y P=\left(l x^{2 l}+m y^{2 m}\right)\left(z-z_{0}\right)^{\alpha_{k}} H_{1}^{k} .
$$

then from Lemma 7.(b) it follows that $\mathcal{X}$ has $k$ invariant planes of the form $a x+b y$ because $l x^{2 l}+m y^{2 m}$ is irreducible, hence $\mathcal{X}$ has $2 k$ invariant meridians. This complete the proof of statement (c).

To finish the proof of theorem we will provide the proof of statement (d).

If we consider $H_{1}^{0}=1$ in $\mathcal{X}$, then $\mathcal{X}$ does not have any invariant meridian because $\mathcal{E}_{\{x, y\}}(\mathcal{X})$ has no factors of the form $a x+b y$. Therefore every $\nu \in\{0,1,2, \ldots, 2(d-2 n)\}$ we can choose $\mathrm{H}_{2}$ in such a way that $\mathcal{X}$ has $\nu$ different invariant parallels which are periodic orbits since $\mathcal{X}$ does not have any singular point on $\mathbb{T}^{2}(l, m, n)$. In addition, these invariant parallels are stable or unstable (algebraic) limit cycles because the sign of $R$ is constant between two consecutive parallels and it changes when we cross one parallel. 
If we consider $H_{2}=1$ in $\mathcal{X}$ then $\mathcal{X}$ does not have any invariant parallel because $z-z_{0}$ is no a factor of $R$ for any constant $z_{0}$. Therefore $\mathcal{X}$ has $2 k$ different invariant meridians which are periodic orbits since $\mathcal{X}$ does not have any singular point on $\mathbb{T}^{2}(l, m, n)$. On the other hand the differential system associated to $\mathcal{X}$ in cylindrical coordinates satisfies that

$$
\dot{\theta}=\frac{x Q-y P}{r^{2}}=\left(\frac{\left.l r^{2 l} \cos ^{2 l} \theta+m r^{2 m} \sin ^{2 m} \theta\right)}{r^{2}}\right)\left(z-z_{0}\right)^{\alpha_{k}} H_{1}^{k}(r \cos \theta, r \sin \theta) .
$$

Since $\left|z_{0}\right|>1$, between two consecutive invariant meridians the

$$
\operatorname{sign}(\dot{\theta})=\operatorname{sign}\left(H_{1}^{k}(r \cos \theta, r \sin \theta) \in\{-1,1\},\right.
$$

and these signs change when we cross one meridian. So the periodic orbits on the invariant meridians are stable or unstable (algebraic) limit cycles alternately.

Proof of Lemma 11. From Lemma 13, the polynomial vector field (24) is an element of $\mathcal{R}_{d}$, so $\mathcal{R}_{d} \neq \emptyset$. As the number of free parameters in $H_{2}$ is $d-2 n+1$ then $\mathcal{R}_{d}$ can be identify with a real linear space of dimension at least $d-2 n+1 \geq 1$.

On the other hand, recall that $\mathfrak{X}_{d}(l, m, n)$ can be identified with $\mathbb{R}^{q}$, for some $q \geq 1$. The previous argument implies that $q \geq d-2 n+1 \geq 1$ On the other hand, $R(0,0, z)$ is a polynomial in $\mathbb{R}[z]$ whose coefficients are linear combinations of the coefficients of $R$, then the subset $\mathfrak{X}_{d}(l, m, n) \backslash \mathcal{R}_{d}$ of polynomial vector fields such that $R(0,0, z) \equiv 0$ has codimension 1 in $\mathfrak{X}_{d}(l, m, n)$. Therefore $\mathcal{R}_{d}$ is generic.

Proof of Lemma 12. First we prove that $\mathcal{G}_{d} \neq \emptyset$. We are assuming that $d \geq 2 l-1$. Then we consider an arbitrary homogeneous polynomial $H_{1} \in \mathbb{R}[x, y]$ of degree $d-2 l+1$, which has $d-2 l+2$ independent real coefficients. It is easy to see that the polynomial vector field

$$
\mathcal{X}=\left(-m y^{2 m-1} H_{1}\right) \frac{\partial}{\partial x}+\left(l x^{2 l-1} H_{1}\right) \frac{\partial}{\partial y}+0 \frac{\partial}{\partial z},
$$

is of degree $d$ and satisfies (6) with $K \equiv 0$. Thus $\mathcal{X} \in \mathfrak{X}_{d}(l, m, n)$. For this vector field $P_{d}=-m y^{2 m-1} H_{1}$ and $Q_{d}=l y^{2 l-1} H_{1}$, hence

$$
G_{d}=x Q_{d}-y P_{d}=\left(l x^{2 l}+m y^{2 m}\right) H_{1} \not \equiv 0 .
$$

This implies that $\mathcal{X} \in \mathcal{G}_{d}$. So $\mathcal{G}_{d}$ can be identify with a real linear space of dimension at least $d-2 l+2 \geq 1$.

On the other hand, recall that $\mathfrak{X}_{d}(l, m, n)$ can be identified with $\mathbb{R}^{q}$, for some $q \geq 1$. The previous argument implies that $q \geq d-2 l+2 \geq 1$ On the other hand, $G_{d}=x Q_{d}-y P_{d}$ is a polynomial in $\mathbb{R}[x, y]$ whose coefficients are linear combinations of the coefficients of $P_{d}$ and $Q_{d}$, then the subset $\mathfrak{X}_{d}(l, m, n) \backslash \mathcal{G}_{d}$ of polynomial vector fields such that $G_{d}=x Q_{d}-y P_{d} \equiv 0$ has codimension 1 in $\mathfrak{X}_{d}(l, m, n)$. Therefore $\mathcal{G}_{d}$ is generic.

Proof of Lemma 13. In is easy to see that $\mathcal{X}$ satisfies (6) by taking $K=4 \ln z^{2 n-1} H_{2}$. Now we will see that $\mathcal{X}$ is of degree $d$ in both cases: $l=m$ and $l>m$.

Case 1. $l=m$. Hence $\operatorname{deg}(P)=\operatorname{deg}(Q)=d$. In this case $R=2 l\left(z^{2 n}-1-r^{2} f\right) H_{2}$ then $\operatorname{deg}(R)=\max \{2 n, 2 l\}+d-2 n$ and since $2 n \geq 2 l, \operatorname{deg}(R)=d$. Therefore $\mathcal{X} \in \mathfrak{X}_{d}(l, l, n)$.

Case 2. $l>m$. Hence $\operatorname{deg}(P)=\operatorname{deg}(Q)=d$ and $\operatorname{deg}(R)=\max \{2 n, 2 l+2 m\}+d-2 n$, as in this case $2 n \geq 2 l+2 m$ then $\operatorname{deg}(R)=d$. Therefore $\mathcal{X} \in \mathfrak{X}_{d}(l, m, n)$.

From the previous cases we conclude that $\mathcal{X} \in \mathfrak{X}_{d}(l, m, n)$. In addition we have that $R(0,0, z)=2 l\left(z^{2 n}-1+r^{4}\right) H_{2} \not \equiv 0$ and $G=x Q-y P=\left(l x^{2 l}+m y^{2 m}\right)\left(z-z_{0}\right)^{\alpha_{k}} H_{1}^{k}$, thus $G_{d}=\left(l x^{2 l}+m y^{2 m}\right)\left(-z_{0}\right)^{\alpha_{k}} H_{1}^{k} \not \equiv 0$. In conclusion $\mathcal{X} \in \mathcal{R}_{d} \cap \mathcal{G}_{d}$. 
Acknowledgments. The first author is partially supported by the MICIIN/FEDER grant number MTM2008-03437, by the AGAUR grant number 2009SGR 410, and by ICREA Academia.

\section{REFERENCES}

[1] Artés, J.C., Grünbaum, B., Llibre, J.: On the number of invariant straight lines for polynomial differential systems, Pacific J. of Math. 184, 207-230 (1998).

[2] Camacho, M.I.T.: Geometric properties of homogeneous vector fields of degree two in $R^{3}$, Trans. Amer. Math. Soc 268, 79-101 (1981).

[3] Christopher, C.: Invariant algebraic curves and conditions for a centre, Proc. Roy. Soc. Edinburgh Sect. A 124, no. 6, 1209-1229 (1994).

[4] Christopher, C., Llibre, J., Pereira, J.V.: Multiplicity of invariant algebraic curves in polynomial vector fields, Pacific J. Math. 229, 63-117 (2007).

[5] Darboux, G.: Mémoire sur les équations différentielles algébriques du premier ordre et du premier degré (Mélanges), Bull. Sci. math. 2ème série 2, 60-96; 123-144; 151-200 (1878).

[6] Darboux, G.: De l'emploi des solutions particulières algébriques dans l'intégration des systèmes d'équations différentielles algébriques, C. R. Math. Acad. Sci. Paris 86, 1012-1014 (1878).

[7] Dobrovol'ski, V.A., Lokot', N.V., Strelcyn, J.M.: Mikhail Nikolaevich Lagutinskii (18711915): un mathématicien méconnu, (French) [Mikhail Nikolaevich Lagutinskii (1871-1915): an unrecognized mathematician] Historia Math. 25, 245-264 (1998).

[8] Hilbert, D.: Mathematical problems, Bull. Amer. Math. Soc. 8, 437-479 (1902); reprinted, Bull. Amer. Math. Soc. (N.S.) 37, 407-436 (2000).

[9] Ilyashenko, Y.: Centennial history of Hilbert's 16th problem, Bull. Amer. Math. Soc. 39, 301-354 (2002).

[10] Lansun, C., Xinan, Z., Zhaojun, L.: Global topological properties of homogeneous vector fields in $\mathbb{R}^{3}$, Chin. Ann. of Math. 20, 185-194 (1999).

[11] LI, J.: Hilbert's 16th problem and bifurcations of planar polynomial vector fields, Internat. J. Bifur. Chaos Appl. Sci. Engrg. 13 , 47-106 (2003).

[12] Jounnolou, J. P.: Équations de Pfaff algébriques. Lecture Notes in Mathematics, Vol. 708. Springer, Berlin, (1979).

[13] Llibre, J., Medrado, J. C.: On the invariant hyperplanes for d-dimensional polynomial vector fields, J. Phys. A: Math. Theor. 40, 8385-8391 (2007).

[14] Llibre, J., Medrado, J. C.: Limit cycles, invariant meridians and parallels for polynomial vector fields on the torus. Bull. Sci. Math. 135 (2011), no. 1, 1-9.

[15] Llibre, J., Pilyugina, V.: Number of invariant straight lines for homogeneous polynomial vector fields of arbitrary degree and dimension. J. Dynam. Differential Equations 21, no. 3, 487-499 (2009).

[16] Llibre, J., PessoA, C.: Invariant circles for homogeneous polynomial vector fields on the 2dimensional sphere, Rend. Circulo Mat. di Palermo 55, 63-81 (2006).

[17] Llibre, J., Rodríguez, G.: Invariant hyperplanes and Darboux integrability for d-dimensional polynomial differential systems, Bull. Sci. Math. 124, 599-619 (2000).

[18] Llibre, J., Zhang, X.: Darboux theory of integrability in $\mathbb{C}^{n}$ taking into account the multiplicity. J. Differential Equations 246, no. 2, 541-551 (2009).

[19] Pereira, J.V.: Vector fields, invariant varieties and linear systems, Ann. Inst. Fourier (Grenoble) 51, 1385-1405 (2001).

[20] Sokulski, J.: On the number of invariant lines of polynomial vector fields, Nonlinearity 9, 479-485 (1996).

[21] Guanguian, S., Jifang, S.: The $n$-degree differential system with $(n-1)(n+2) / 2$ straight line solutions has no limit cycles, in "Proc. of Ordinary Differential Equations and Control Theory", Wuhan (in Chinese), 216-220 (1987).

[22] Zhang, X.: Number of integral lines of polynomial systems of degree three and four, J. of Nanjing University, Math. Biquartely 10, 209-212 (1993).

[23] ZHANG, X.: Invariant hyperplanes and Darboux integrability of polynomial vector fields, J. Phys. A: Math. Gen. 35, 9931-9941 (2002). 